\title{
Iterative Threshoding and Morphology Operation based Melanoma Image Segmentation
}

\author{
Abbas Hussien Miry \\ Electrical Engineering Dept. \\ AL-Mustansiriyah University
}

\begin{abstract}
Dermoscopy is a suitable diagnostic technique for biology observation of pigmented skin lesions used in dermatology. Nowdays there is great interest in the prospects for methods of automatic image analysis for dermoscopy image, it Provide quantitative information about the lesion, which can be of link the doctor, and as a standalone early warning tool. This paper presents a good method of melanoma images segmentation. It based on threshoding as segmentation and mathematical morphology used to remove unwanted part in order to obtain a better segmentation. The proposed method is compared with the famous method of segmentation of skin lesions in images dermoscopic such adaptive thresholding and fuzzy K-means clustering for the segmentation and evaluated with two metrics, False Positive Rate (FPR) and the False Negtive Rate (FNR), using the segmentation results obtained by a dermatologist experienced and ground truth.
\end{abstract}

\section{Keywords}

Morphology Operation, images segmentation, skin lesions.

\section{INTRODUCTION}

Melanoma is a type of skin cancer. It is one of the most dangerous diseases which lead to death. Dermoscopy is useful for dermatologists in distinguishing the lesion from the surface of the earth which are non-cancerous, particularly to detect skin cancer. Malignant melanoma, which is a serious type of skin cancer and is also found in the bowel and the eye. It is scheduled to uncontrolled growth of pigment cells. There is no other tests for the diagnosis of malignant melanomas. At the present time and visual diagnosis of melanomas is the most common method, which works by trained professionals [1].

This method allows the recognition of the formal structures are not visible to the naked eye, which opens a new dimension of clinical morphological features of pigmented skin lesions. In the past few years there have been significant developments in each of dermoscopy and telemedicine, which allows improving the clinical diagnosis of skin lesions. At present there is great interest in the chances of having an early screening system for teledermatoscopy, based on an automated analysis of the images dermatoscopic. The benefits of these systems are twofold: (1 ) to provide quantitative information on the lesion that could be relevant to the doctor, (2 ) to be used as a standalone tool early warning, with the inherent advantages of the effectiveness of time and procedures low-cost diagnosis and treatment [2].

Usual approach in a dermoscopic image analysis mechanism usually has some step such segmentation, feature extraction and selection and classification of the lesion. Image segmentation is one of the most important because it affects the accuracy of the subsequent steps. However, it is difficult to segmentation due to a large variety of lesion shapes, sizes, and colors along with different skin types and textures. In addition, some of the lesions with irregular borders, and in some cases there is a smooth transition between the lesion and the skin. Other difficulties are related to the presence of dark hair covering the lesions and the presence of mirror reflections [3].

While several individual techniques have been proposed for the segmentation of lesions in dermoscopic images, [2] study a comparison of segmentation methods applied to set of dermoscopic image analysis, along with a clinical evaluation of each segmentation result performed by an experienced dermatologist.

Six methods for the segmentation of skin lesions in dermoscopic images were presented in [3], this set includes some state of the art techniques which have been successfully used in many medical imaging problems and the level set method. [1] segmenting the dermoscopic image using the machine learning algorithms. The algorithms such as Back Propagation network (BPN), Radial Basis Function Network (RBF) and Extreme Learning Machine (ELM) were used.

In this paper we propose and evaluate segmentation methods for dermoscopic images. It based on histogram as segmentation and mathematical morphology used to remove unwanted part in order to obtain a better segmentation.

\section{ADAPTIVE THRESHOLDING}

In the local adaptive technology, the threshold is calculated for each pixel, based on local statistics such as range, contrast, or bone surface parameters of the pixel neighborhood. It can be approached in different ways such as background subtraction and model the flow of water, which means the standard derivation of pixel values, in contrast to the local image. Some of the drawbacks of local techniques threshold level are dependent area size the characteristics of individual image, and time consuming. Therefore, some researchers have used a hybrid approach that applies both methods the threshold level of global and local and some morphological operators to use.

Niblack[4], use local variation technique. In this methods, the minimum is calculated based on the average local and standard deviation within the framework [4].

He calculates the threshold pixel wise by moving the window over the image gray level. The calculation of the threshold is based on the mean $a v$ and local standard deviation of each pixel in the window is given below:

$T_{\mathrm{AT}}=a v+\sqrt{\frac{\sum p i^{2}}{N P}-a v^{2}}$ 
Where NP is the number of pixels in the image gray, av is the average pixel value $p i$ is fixed to -0.2 by the [5]. The benefit of Niblack is it always identifies areas of the text correctly as the lead but on the other hand tends to produce a large amount of noise binarization in non-text areas also [5].

\section{FUZZY K -MEAN CLUSTERING ALGORITHM}

One of the models most commonly used is the fuzzy K-means (FKM). FKM algorithm assigns members that are inversely associated with the relative distance from a point to the models that are cluster centers in the FKM Model [6].It is a way to assemble and which allows a single data point belong to two or more groups with different membership grade. Often this method is used in pattern recognition. It is based on minimizing the following objective function

$J_{m}=\sum_{i=1}^{N} \sum_{j=1}^{k} u_{i j}^{m}\left\|X_{i}-K_{j}\right\|^{2} \quad 1 \leq m \leq \alpha$

where $m$ is any real number greater than $1, u_{i j}$ is the degree of membership of $X_{i}$ in the cluster $j, X_{i}$ is the $i$ th of ddimensional measured data, $K_{j}$ is the d dimension center of the cluster, and $\|*\|$ is any norm expressing the distance between any measured data and the center [7].

\section{PROPOSED METHOD}

Suppose that given dermoscopy image as shown in Fig.1 ,while Fig. 2 shows the segmented image by medical expert , and required to segment the lesion region ,the proposed method contains the following stages:

1) Thresholding

Suppose we have image $\mathrm{I}(\mathrm{x}, \mathrm{y})$. The number of pixels with gray level $\mathrm{i}$ is denoted $\mathrm{h}_{\mathrm{i}}$, Then, the threshold $T H$ that divides the range into two classes which are $\mathrm{H} 1=[1, \ldots, T H]$ and $\mathrm{H} 2=[T H+1, \ldots, \mathrm{L}]$. Then, the gray level histogram for the two classes is:

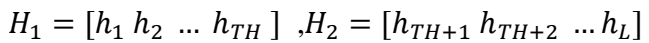

Also, the median for classes $H_{1}$ and $H_{2}$ are $m d_{1}, m d_{2}$ respectively, let $m_{T}$ be the overall median of the whole image.

$m d_{T}=\frac{m d_{1}}{m d_{2}}$

in next step, the threshold $T H$ is equal to $m d_{T}$ and divied the result image to two new group $H_{1}$ and $H$.repeat this process untile no change in value of $m d_{T}$. Fig. 3 shows the segment images after this step.

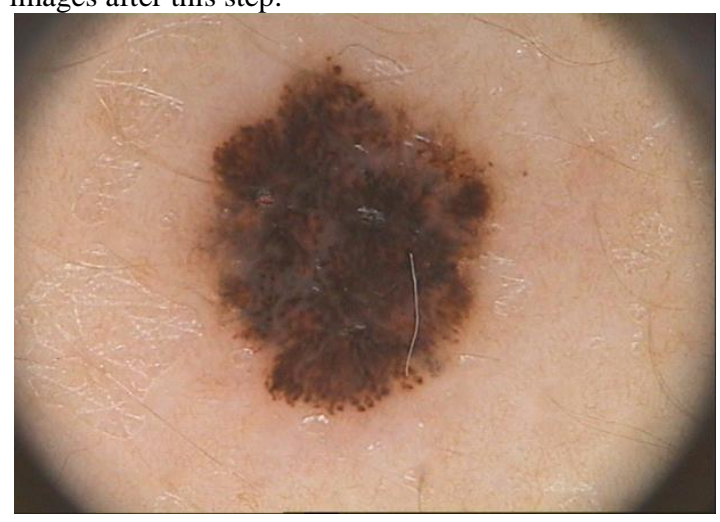

Fig.1 Original image

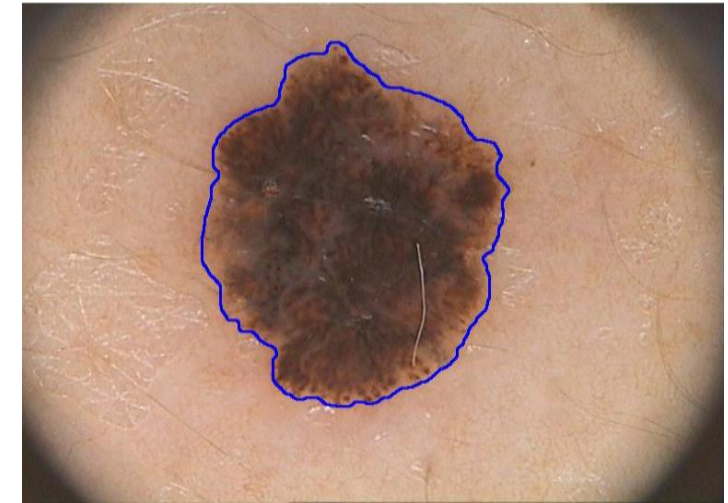

Fig.2 Segmentation obtained by a medical expert

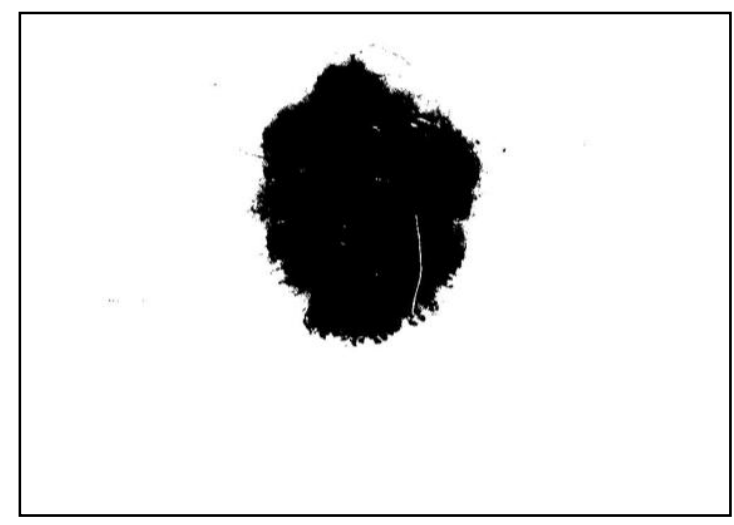

Fig.3 Segmentation mask obtained by threshoding step

2) Morphology operation

After threshoding step it required to element unwanted point such noise to implement best segmentation it is required to use morphology operation.

Mathematical morphology is the name for a group of operators based on set theory and the definition of abstract structure known as the infinite lattice. Morphological operators include erosion, dilation, opening and closing, rank candidates (including filters and medium), transfers hat, and other derivative transfers. This can be defined operations on binary images or gray in any number of dimensions. It can also be specified with Euclidean (isotropic) or non-Euclidean metrics (geodesic). The main areas of application of mathematical morphology tools are medical imaging, materials science and machine vision, where morphological transformations are particularly useful for image analysis and image enhancement [8].

Morphological processes such as erosion, dilation, opening, and closing. Often use combinations of these operations to analyze morphological picture. There are many useful operators in the definition of mathematical morphology. They are stretched, corrosion, and opening and closing [9].

The application of morphological operations structuring element to the image input, output and create an image of the same size. In the process of formality, and is based on the value of each pixel in the image output comparison between the corresponding pixel in the input image with its neighbors. By choosing the size and shape of the neighborhood, you can build a morphological process that is sensitive to the specific forms in the input image [9]. 
Dilation: the value of the output pixel is the maximum value of each pixel in the neighborhood of the input pixel. In binary form, if any set of pixels to a value of 1, the output pixel is set to 1 .

Erosion: the output pixel value is the minimum value of each pixel in the neighborhood of the input pixel. In binary form, if any set of pixels to 0 , the output pixel is set to 0 .

Fig. 1 shows the following extended binary image. Notice how the structuring element determines the neighborhood of the pixel of interest, which flew. Function extends the appropriate rule applies to the pixel in the neighborhood and assigns the value of the corresponding pixel in the output image. In the figure, the morphological dilation function determines the value of the output pixel to 1 because one of the elements in the neighborhood defined by the structuring element.

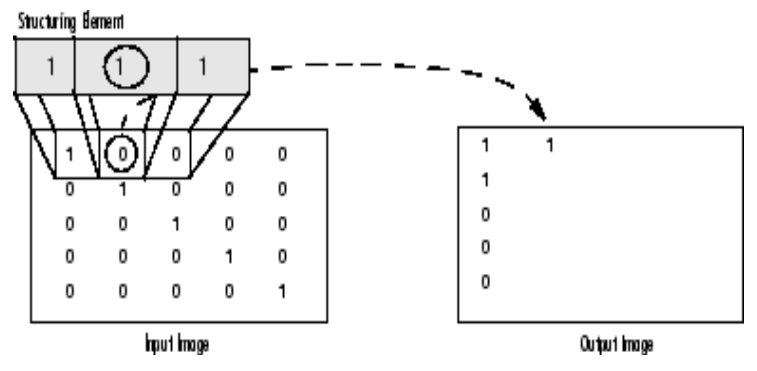

Fig.4 Morphological Dilation of a Binary Image

Figure. 2 shows the processing time for a grayscale image and the processing pixel particularly in the input image. Notice how the function applies to rule on the neighborhood pixel input and uses the highest value of each pixel in the neighborhood where the value of the corresponding pixel in the image output [9].

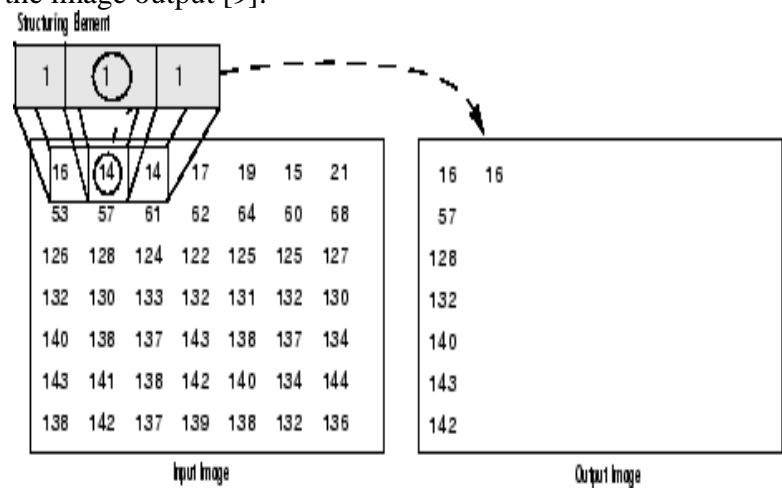

Fig.5 Morphological Dilation of a gray scale image

Mathematical morphology is a powerful tool to deal with the various problems in image processing and computer vision. Morphological operations, which are erosion, dilation, opening and closing are used to extract, edit and manipulate existing features in the image on the basis of its forms. The dilation and erosion of a gray-level image by a two dimensional point set are defined respectively as:-

$(g \oplus B)(r, c)=\max \{g(r-k, c-l) \mid(k, l) \in B)\}$

$(g \ominus B)(r, c)=\max \{g(r+k, c+l) \mid(k, l) \in B)\}$

Opening (closing) is a sequential combination of erosion (dilation) and dilation (erosion).Although the restructuring takes care of the form element through image processing features, it cannot; however, treat objects of the same shape but different size alike. Thus, the processing objects based on their shape, as well as the size of the second merge attribute to the structuring element which is the size. A structuring element along with its homothetic highest ranking features image processing on the basis of the shape as well as size. And called such types of morphological operations as multistage conformation. Is defined as the opening and closing, respectively[10], as:-

$$
\begin{aligned}
& (g \circ B)(r, c)=((g \ominus n B) \oplus n B)(r, c) \\
& (g \bullet B)(r, c)=((g \oplus n B) \ominus n B)(r, c)
\end{aligned}
$$

the result from provirus stage is filtered with a filter close using a morphological structuring element in order to remove features from the image, the dark areas has been removed in the four corners of the images as shown in Fig.6 .Finally the segment image shown in Fig.7

\section{SIMULATION RESULTS}

This method has been tested on a 40 color images dermoscopy Spanish Pedro Hospital [11]. This is the colorful pictures (RGB) with 24 bits per pixel and 764 x574 pixel spatial Decision. The results were compared with the segmentation Those of a standard algorithm.

Different parameters were used to analyze the performance of various methods. They are False Positive Error (FPE), False Negative Error (FNE) and True Detection Rate (TDR) Coefficient of similarity and spatial overlap[12]. To define the first two types of quality metrics let SR denote the result of an automatic segmentation method and GT denote the ground truth segmentation obtained by the medical expert. Both SR and GT are binary images such that all the pixels inside the curve have label 1 and all others have label 0 . The metrics are calculated as follows:

False positive rate (FPR)

This metric measures the rate of pixels classified as lesion by the automatic segmentation that were not classified as lesion by the medical expert:

$F P R(S R, G T)=\frac{A(S R \cap \overline{G T})}{A(G T)}$
False Negative Rate (FNR)

This metric measures the rate of pixels classified by the medical expert as lesion that were not classified as lesion by the automatic segmentation:

$\operatorname{FNR}(X, Y)=1-\frac{A(S R \cap G T)}{A(G T)}$

True Detection Rate (TDR)

This metric measures the rate of pixels classified as a scourge by the entire automatic segmentation medical expert

$\operatorname{TDR}(X, Y)=\frac{\neq(X \cap Y)}{\neq(Y)}$

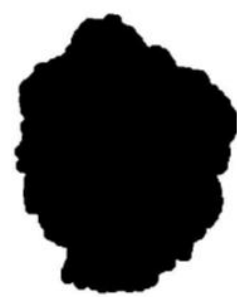

Fig.6 Segmentation mask obtained by a proposed method 


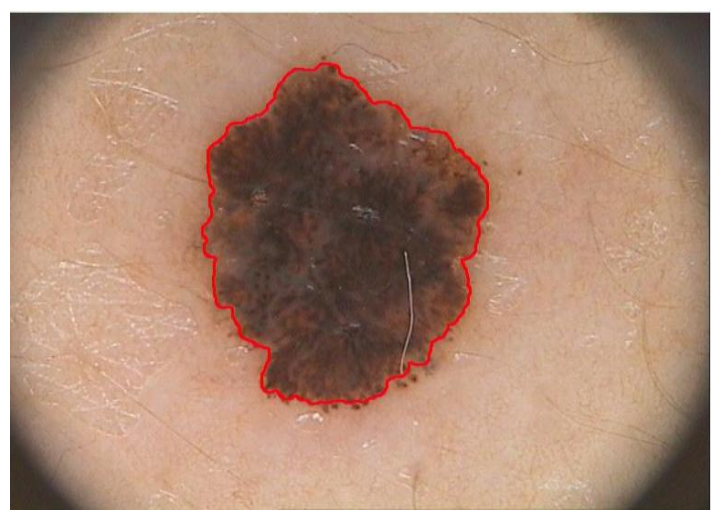

Fig.7 Segmentation obtained by the proposed method

Database has been processed images using all methods and assessment agent. The results were compared with the reference image fragmentation (the facts on the ground) and segmentation errors were evaluated by three measures mentioned above. Table 1 shows the average scores for each method..

Table .1 Results of test images for different methods

\begin{tabular}{|c|c|c|c|}
\hline Method & FNR (\%) & FPR (\%) & TDR(\%) \\
\hline AT & 16.45 & 7.20 & 83.2 \\
\hline FKM & 6.17 & 5.38 & 92.6 \\
\hline ML & 9.1 & 2.1 & 94.7 \\
\hline Proposed & 3.6 & 1.62 & 96.2 \\
\hline
\end{tabular}

The best way according to FPR is proposed with $1.62 \%$ in term of FPR the best result found in proposed method Fig. 8 shows the segmentation of different methods

\section{CONCULSIONS}

A way of segmentation is proposed with the process of morphological, and it is capable of real part images of medical examinations. Morphological operation help to reject unwanted object in segmented image, also the method of segmentation is very simple and has a better result, These images were manually segmented by an expert dermatologist and classified the type of lesion were compared to the output of the proposed segmentation methods with segmentation images manually using two different measures (FPR and the FNR). This technique is found it to be better than other contemporary methods, both in terms of FPR and the FNR. This approach can be used to support decision-making problems in medical diagnosis. The proposed methods can be used for segmentation of the pest in aided diagnostic computer system to assist the clinical diagnosis of dermatologists. In a future it can be used optimization algorithm such genetic or PSO to find optimal value of thresholding with fitness function equal to the error between expert and proposed method.

\section{REFERNCE}

[1] G.S.Vennila, L.P. Suresh and K.L.Shunmuganathan," Dermoscopic Image Segmentation and Classification using Machine Learning Algorithms", International Conference on Computing, Electronics and Electrical Technologies,pp: 1122- 1127,2012.

[2] T. Mendonca, A. R. S. Marca, A. Vieira ,J. C. Nascimento, M. Silveira, J. S. Marques and J. Rozeira, "Comparison of Segmentation Methods for Automatic Diagnosis of

[3] Dermoscopy Images", Proceedings of the 29th Annual International Conference of the IEEE EMBS , France ,pp: 6572- 6575 , August, 2007.(IVSL)

[4] M. Silveira, J. C. Nascimento, J. S. Marques,A.R. S. Marçal, T. Mendonça, S.Yamauchi, J. Maeda, and J. Rozeira ,"Comparison of Segmentation Methods for Melanoma Diagnosis in Dermoscopy Images ",IEEE Journal of Selected Topics in Signal Processing, Vol. 3, No. 1,pp:35-45 February 2009.

[5] T.R. Singh , S. Roy, O.I. Singh, T. Sinam and K.M. Singh"A New Local Adaptive Thresholding Technique in Binarization", IJCSI International Journal of Computer Science, ISSN : 1694-0814, Issues, Vol. 8, Issue 6, No 2,pp:271-277, 2011.

[6] R. Saini and M. Dutta ," Image Segmentation for Uneven Lighting Images using Adaptive Thresholding and Dynamic Window based on Incremental Window Growing Approach", International Journal of Computer Applications (0975 - 8887) Vol. 56, No.13, pp: 31 -36 , 2012.

[7] N. R. Pal, K. Pal, J. M. Keller, and J. C. Bezdek," A Possibilistic Fuzzy c-Means Clustering Algorithm", IEEE Transactions on Fuzzy Systems, Vol.. 13, No. 4,pp:517-530, August 2005.

[8] S. Poddar and A. Mukhopadhayay ," Cluster: A MATLAB GUI Package for Data Clustering" International Technology Research Letters, Vol.1 Issue1,pp:78- 83, 2012.

[9] M. Pesaresi and J. A. Benediktsson ," A New Approach for the Morphological Segmentation of High-Resolution Satellite Imagery",IEEE Transaction on Geosciencs and Remote Sensing, Vol. 39, No. 2,pp:309-320,2001.

[10] A. P. Vartak and V. Mankar, " Morphological Image Segmentation Analysis", International Journal Of Computer Science And Applications Vol. 6, No.2, pp:161-165,2013.

[11] S. Mukhopadhyay and B. Chanda ," Multiscale Morphological Segmentation of Gray-Scale Images", IEEE Transactions on Image Processing, Vol. 12, No. 5,pp:533-549, 2003. http://www2.fc.up.pt/addi/

[12] L. P. Suresh, K.L. Shunmuganathan and S.H. K. Veni ," Dermoscopic Image Segmentation using Machine Learning Algorithm" American Journal of Applied Sciences Vol.8 ,No.11, pp: 1159-1168, 2011 

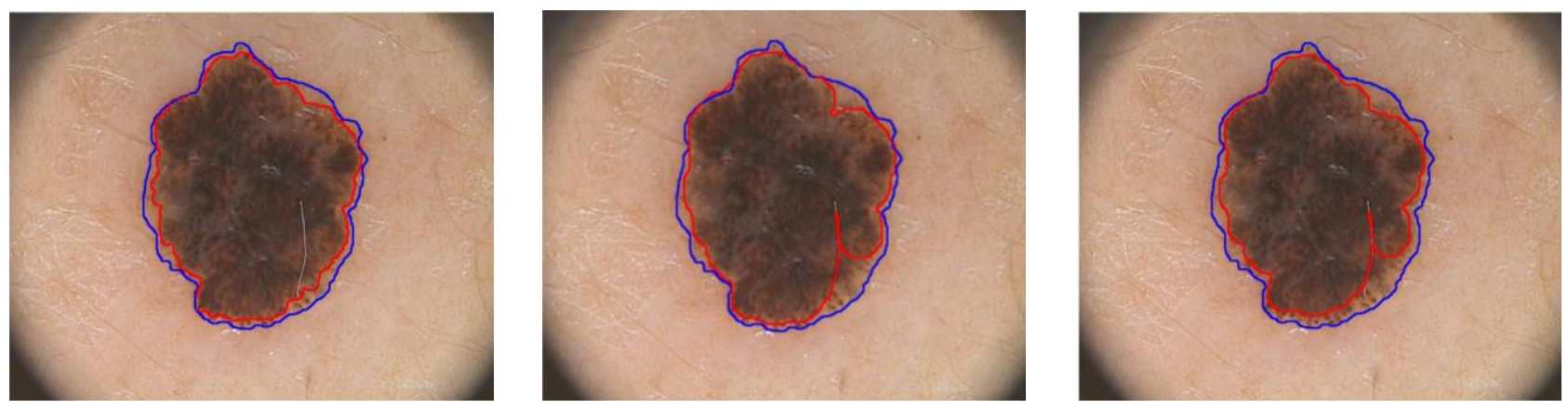

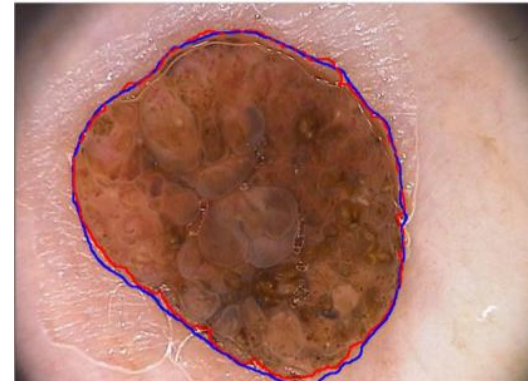

(a)

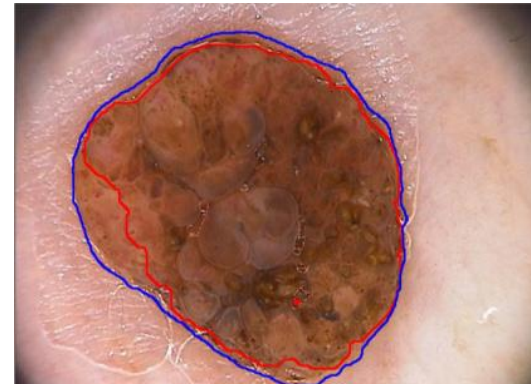

(b)

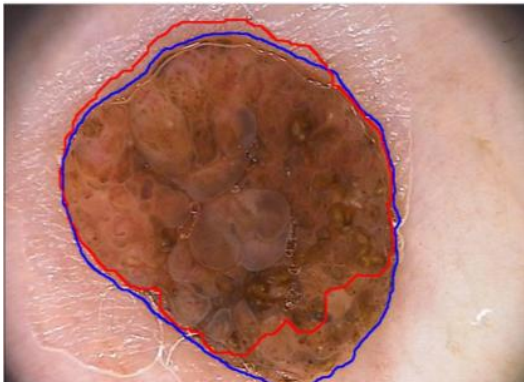

(c)

Fig.8 Segmentation of the dermatoscopic image by: Blue border by expert segemntation : Red border segmentation by (a) proposed method, (b) FKM ,(c)AT 